\title{
Numerical quantification of coupling effects for radiation-conduction heat transfer in participating macroporous media: Investigation of a model geometry
}

\author{
David Y.S. Perraudin, Sophia Haussener* \\ Laboratory of Renewable Energy Science and Engineering, EPFL, Station 9, 1015 Lausanne, Switzerland
}

\section{A R T I C L E I N F O}

Article history:

Received 31 October 2016

Received in revised form 21 March 2017

Accepted 22 March 2017

\section{Keywords:}

Semitransparent porous media

Coupled radiative-conductive heat transfer

Monte Carlo ray tracing

Finite volumes

\begin{abstract}
A B S T R A C T
Radiative-conductive heat transfer in porous media is usually investigated by decoupling the heat transfer modes and solving the volume-averaged continuum equations using effective transport properties. However, both modes are naturally coupled and coupling effects might significantly affect the results. We aim at providing quantitative understanding of the coupling effects occurring in a model geometry. This is an important first step towards improving the accuracy of heat transfer predictions in engineering applications.

We developed a numerical method using a structured mesh, cell centered finite volumes and Monte Carlo ray tracing techniques in order to simulate the 3-dimensional and unsteady coupled radiativeconductive heat transfer in semitransparent macroporous media. We have optimized the numerical method with regards to memory and computational requirements leading to optimal performance and allowing to perform a parameter variation study for various steady state cases.

We conducted a parameter study considering different optical and thermal material properties and boundary conditions in order to quantify the coupling effect between conduction and radiation, and to demonstrate its dependencies. In terms of thermal properties, it was found that the ratio of bulk thermal conductivities is governing the coupling effect. A distinct peak at a given conductivity ratio was found. The influence of optical properties is discussed in details. It was found that a significant coupling effect exists, reaching up to $15 \%$ of the total thermal heat flux.

The verified modeling framework in conjunction with our non-dimensionalization offers a tool to investigate the importance of radiation-conduction coupling in a quantitative manner. It is an important step towards understanding the detailed mechanisms of radiation and conduction coupling and provides engineering guidelines on the importance of these effects.
\end{abstract}

() 2017 Elsevier Ltd. All rights reserved.

\section{Introduction}

Semitransparent porous media are of interest in a variety of applications, including solar energy conversion, space and medical technologies, or chemical processing. The multiple scales present in such applications remain a challenge for engineering. At high temperatures, radiative heat transfer can dominate the heat transfer and certain ceramics that are opaque at room temperatures become transparent. Conversely, at room temperature, without external irradiation, radiative heat transfer is negligible. In the absence of fluid flow, heat transfer occurs by conduction and radiation simultaneously. The term coupled is used to highlight the nat-

\footnotetext{
* Corresponding author.

E-mail address: sophia.haussener@epfl.ch (S. Haussener).
}

urally occurring interaction between radiative and conductive heat transfer. The unsteady heat transfer equation in a homogeneous media accounting for radiation and conduction incorporates, in addition to the divergence of the conductive heat fluxes, also the divergence of the radiative fluxes as a non-linear source term, resulting in the complex interdependence. Additionally in porous media, this equation has to be solved for each homogeneous phase.

The multiscale nature of applications incorporating porous materials makes it often impossible to run direct numerical simulations for heat transfer. Instead, effective radiative and conductive properties of porous media are used in order to allow for efficient simulation. The approach is based on the assumption that the porous media can be approximated by an analogous media consisting of two homogeneous and continuous phases. The properties of these analogous phases, also called effective transport properties, 


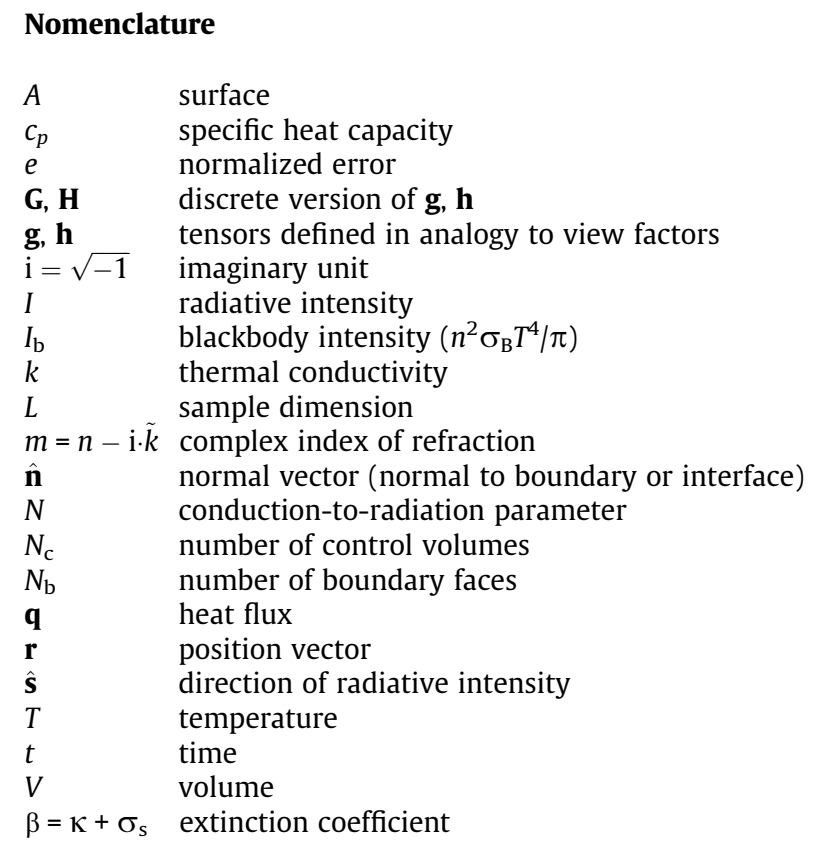

\author{
polar angles \\ absorption coefficient \\ wavelength \\ modified radiation-to-conduction parameter \\ coupling effect \\ density \\ scattering coefficient \\ Stefan Boltzmann constant \\ azimuthal angle \\ scattering phase function \\ porosity \\ solid angle
}

$\begin{array}{ll}\text { Subscripts } & \\ \mathrm{s} & \text { solid phase } \\ \mathrm{f} & \text { fluid phase } \\ \mathrm{r} & \text { radiative } \\ \mathrm{e} & \text { emitted } \\ \mathrm{b} & \text { boundary } \\ i, j & \text { iteration parameters }\end{array}$

are determined such that the solution of the homogenized problem results in the same temperature or intensity fields as the solution to the original, discrete-scale problem. This approach allows for efficient computations. Details of volume averaging theory applied to problems relevant in heat and mass transfer are described in [1]. Effective properties can be determined experimentally [2,3] or through simulations. As the effective properties significantly depend on the geometry of the porous media, accurate computational approaches directly incorporate the exact morphology using, for example, computed tomography of the materials of interest [48]. There have been attempts to summarize effects of conduction and radiation into one single parameter sometimes referred to as "phononic diffusivity" $[9,10]$ or "equivalent conductivity" [11]. This parameter must, by definition, heavily depend on temperature.

The interest in understanding the radiative-conductive coupling in semitransparent porous media comes from the fact that various models have been developed for the separate determination of the effective radiative [12] and effective conductive [13] properties but their application in the coupled case is ambiguous. Superimposing the effect of radiation and conduction in order to obtain a solution to a coupled problem seems convenient but not necessarily accurate. The aim of this work is to study, whether the superposition of conductive and radiative heat fluxes computed separately is a valid procedure, or whether coupling effects exist, and to quantify their sensitivity to bulk material properties. In case superposition is justified, existing models can easily be combined. In case coupling effects exist, their quantification will allow using existing models, improved with a well investigated and quantified coupling effect.

Theoretical work has predicted the existence of coupling effects [14]. This work is based on the derivation of the volume-averaged energy equations for porous media and shows that in the volumeaveraged equations additional coupling terms for radiation and conduction exist. However, no quantification is given for a realistic case and the importance of the different terms under various conditions is not given in [14]. Coquard et al. [11] stated that in the case of "metal or ceramic open cell foams" they have "checked from numerous results obtained on different cellular structures with various optical properties that this coupling is relatively weak". Their findings suggest that for some setups the superposition of the results obtained for the two modes separately is justified without giving more details.

We aim at quantifying these coupling effects, at predicting their dependence on boundary conditions and geometrical and material properties. We therefore developed a numerical method which is capable of pore-scale simulations with coupled radiativeconductive heat transfer in macroporous media. Such a method must be significantly more powerful than commonly used methods in single phase setups in one or two dimensions, such as discrete ordinate methods [15], finite elements [16] or spherical harmonics methods ( $\mathrm{P}_{\mathrm{N}}$ method) $[17,18]$. It must be capable of capturing three dimensions, different phases, resolving porous structures, while remaining computationally efficient and accurate. We present results obtained for a model geometry. Structured, lattice-type porous media are of interest in a wide variety of applications (porous burners, heat exchangers, or lightweight structures), provide interesting test media with well-defined structures, and can easiest be implemented as materials by design [19].

\section{Governing equations}

\subsection{Assumptions}

The steady state case of a macroporous media consisting of two phases (for example one fluid and one solid) is considered. Both phases are assumed at rest, such that heat is transferred either by conduction or by radiation. Macroporous implies that the radiative heat transfer occurs in the geometric optics regime such that $\pi L / \lambda \gg 1$ holds if $L$ is a characteristic length scale and $\lambda$ the wavelength. The fluid phase is transparent and the solid phase semitransparent, thus participating in the radiative heat exchange through absorption and internal emission. Internal scattering in the bulk material of the participating phase is neglected $\left(\sigma_{s}=0\right)$. The participating medium and the domain boundaries are grey, such that wavelength dependencies can be dropped. The partici- 
pating material can in that case be described by its optical thickness $\kappa L$ where $\kappa$ is the absorption coefficient and $L$ the sample size. The materials of the two phases are assumed homogeneous and isotropic. Thus the spatial dependencies will not be introduced in the equations given in the following. All material properties are assumed to be independent of temperature. The setup is illustrated in Fig. 1. The relevant bulk material properties for the steady case are the thermal conductivities, $k_{\mathrm{f}}$ and $k_{\mathrm{s}}$, and the complex indices of refraction, $m_{\mathrm{f}}$ and $m_{\mathrm{s}}$. In the case of the fluid phase, the imaginary part of $m_{\mathrm{f}}$ vanishes. A reference wavelength of $\lambda=1 \mu \mathrm{m}$ is used throughout the paper. The relation between the complex part of the index of refraction, $\tilde{k}$, the wavelength, $\lambda$, and the absorption coefficient is given by $\kappa=4 \pi \tilde{k} / \lambda$.

\subsection{Heat transfer equation}

The transient, coupled radiative-conductive heat transfer in a homogeneous medium is a highly non-linear problem and described by Eqs. (1a) and (1b). The effect of conductive heat transfer is included as well as the effect of radiative heat transfer, the latter by means of the divergence of the radiative heat fluxes, $\nabla \cdot \boldsymbol{q}_{\mathrm{r}, i}$. In the transparent phase the divergence of radiative heat fluxes vanishes by definition.

$\rho_{\mathrm{f}} c_{p, \mathrm{f}} \cdot \frac{\partial T_{\mathrm{f}}}{\partial t}=\nabla \cdot\left(k_{\mathrm{f}} \nabla T_{\mathrm{f}}\right)$

$\rho_{\mathrm{s}} c_{p, s} \cdot \frac{\partial T_{s}}{\partial t}=\nabla \cdot\left(k_{\mathrm{s}} \nabla T_{\mathrm{s}}\right)-\nabla \cdot \mathbf{q}_{\mathrm{r}, \mathrm{s}}$

\subsection{Radiative heat transfer}

The radiative heat source at a given location $\mathbf{r}$ is obtained by integration of the absorbed radiative intensity across all solid angles minus the emitted radiative intensity at $\mathbf{r}$ as described in Eq. (2) [20].

$-\nabla \cdot \mathbf{q}_{\mathrm{r}}(\mathbf{r})=\kappa \cdot\left(\int_{4 \pi} I\left(\mathbf{r}, \hat{\mathbf{s}}_{\mathrm{i}}\right) d \Omega_{i}-4 \pi \cdot I_{\mathrm{b}}(\mathbf{r})\right)$

The radiative heat flux is obtained from the radiative transfer equation (RTE) [20]. The RTE (Eq. (3)) describes the change of intensity in the direction $\hat{\mathbf{s}}$. Physical effects contributing to the change of intensity are internal emission, absorption and scattering.

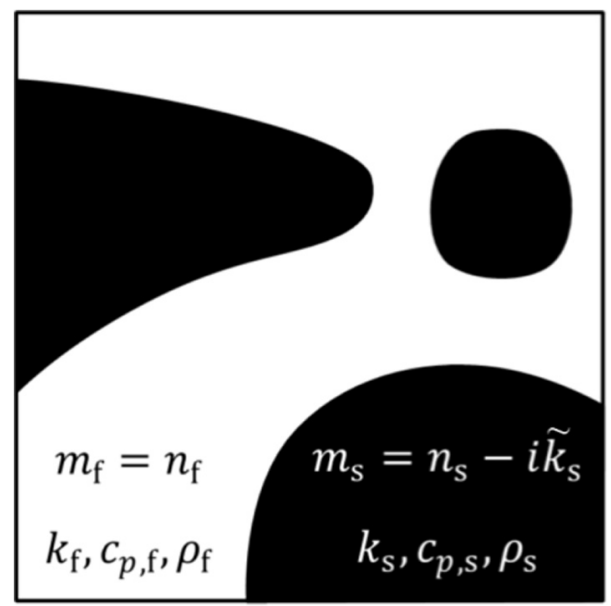

Fig. 1. Illustration of the two-phase setup and the relevant material properties for the transparent fluid and semitransparent solid phase of the macroporous material. $\hat{\mathbf{s}} \cdot \nabla I(\mathbf{r}, \hat{\mathbf{s}})=\kappa I_{\mathrm{b}}(\mathbf{r}, \hat{\mathbf{s}})-\left[\kappa+\sigma_{\mathrm{s}}\right] I(\mathbf{r}, \hat{\mathbf{s}})+\frac{\sigma_{\mathrm{s}}}{4 \pi} \int_{4 \pi} I\left(\mathbf{r}, \hat{\mathbf{s}}_{\mathbf{i}}\right) \Phi\left(\mathbf{r}, \hat{\mathbf{s}}_{\mathbf{i}}, \hat{\mathbf{s}}\right) d \Omega_{i}$

Note that the black body intensity incorporates an $n^{2}$-term. In Eqs. (2) and (3) the wavelength dependency has been dropped, since grey media and surroundings are considered in this study. The relevant properties for an emitting, absorbing and scattering medium are the absorption, $\kappa$, and scattering, $\sigma_{s}$, coefficients and the scattering phase function, $\Phi$. In the present study internal scattering in the bulk solid phase is neglected, such that $\sigma_{\mathrm{s}}$ vanishes and $\Phi$ has no relevance in the equation formulated for the solid phase. Note that considering the radiative heat transfer in the full sample of the macroporous material, scattering effects will still be present due to the material interfaces where reflection and refraction occurs. Some authors $[4,5,12,21]$ have characterized samples of porous media by a scattering coefficient and phase function.

\subsection{Interface condition}

At the interface between phases, continuity of temperatures is enforced. Unlike in the case of a transparent and an opaque phase in contact, no radiative energy is absorbed or emitted at the interface. Radiative energy incident on an interface is either reflected or refracted such that continuity of conductive fluxes must be enforced to guarantee the conservation of energy. In summary, Eqs. (4) and (5) apply.

$\left.T_{\mathrm{f}}\right|_{\text {interface }}=\left.T_{\mathrm{s}}\right|_{\text {interface }}$

$\left.k_{\mathrm{f}} \nabla T_{\mathrm{f}}\right|_{\text {interface }} \cdot \hat{\mathbf{n}}=\left.k_{\mathrm{s}} \nabla T_{\mathrm{s}}\right|_{\text {interface }} \cdot \hat{\mathbf{n}}$

The laws governing the behavior of radiative intensities at interfaces are given by Fresnel's and generalized Snell's laws of reflection and refraction, respectively [20]. The interface behavior is determined by the complex indices of refraction of the two materials in contact, $m_{\mathrm{f}}$ and $m_{\mathrm{s}}$.

\subsection{Domain boundary conditions}

Two kinds of boundary conditions are required at the domain boundaries such that the problem is well posed. One for the temperature and one for the radiation. A temperature or a conductive heat flux can be set for each boundary. In combination with the radiative properties of the boundaries and prescribed incoming radiation, these boundary conditions allow to simulate a large number of realistic situations. In the present study two kinds of boundary conditions are used: black walls at prescribed temperature and perfectly insulated specular mirrors.

\section{Numerical implementation}

\subsection{Method overview}

The computational domain is discretized using a structured grid of cubes. The method itself is not restricted to structured grids, however unstructured grids are significantly more complex to implement. A finite volume, cell centered approach is chosen to solve for the temperature field. Steady state of the heat transfer equation (Eq. (1)) is reached by explicit Euler time integration. At a given time step, the divergence of radiative and conductive heat fluxes is computed for each control volume. The residual is computed and the temperature values are updated. This is repeated until convergence is reached (Fig. 2). The method is applicable to both, steady and unsteady situations but only steady results are discussed in this study. 


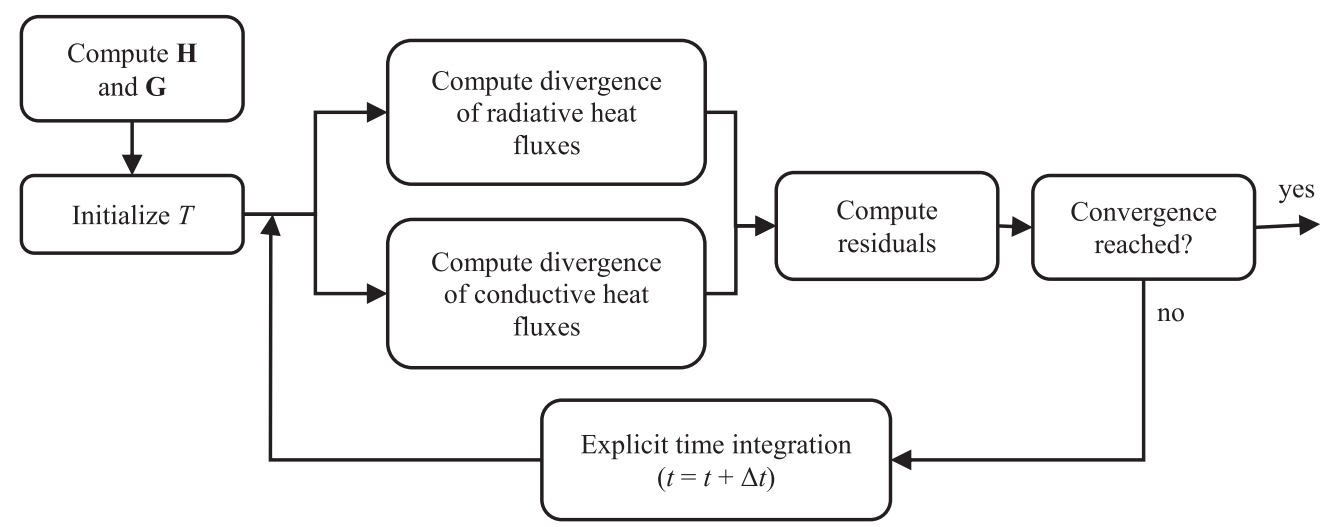

Fig. 2. Flowchart of numerical algorithm used to solve steady state coupled conductive-radiative heat transfer in semitransparent macroporous media.

Note that the cases of conductive heat transfer only ( $\left.\dot{q}_{\text {cond }}\right)$, or radiative heat transfer only $\left(\dot{q}_{\text {rad }}\right)$ can be created artificially by setting the respective heat fluxes to zero. In the presence of both modes, the amount of heat transferred by conduction $\left(\left.\dot{q}_{\text {cond }}\right|_{\text {coupled }}\right)$ and radiation $\left(\left.\dot{q}_{\text {rad }}\right|_{\text {coupled }}\right)$ can be evaluated. This is a key requirement for the investigation of the coupling effect.

\subsection{Conductive heat fluxes}

The divergence of conductive fluxes is computed by performing a balance of the heat fluxes for each control volume. The temperature gradients are computed using second order accurate finite difference stencils for faces that are within the domain and separating two control volumes of the same phase. It follows from Eqs. (4) and (5) that an equivalent conductivity of $\frac{2}{1 / k_{s}+1 / k_{\mathrm{f}}}$ can be used at an interface if the control volumes are of the same size. This equivalent conductivity guarantees continuity of temperatures and heat fluxes. The heat flux at the domain boundaries is computed using a one-sided, first order finite difference stencil.

\subsection{Radiative heat fluxes}

The radiative fluxes are computed using a Monte Carlo ray tracing algorithm. A method proposed in [20] is implemented. Eq. (2) is rewritten as follows (Eq. (6))

$$
\begin{aligned}
\nabla \cdot \mathbf{q}_{\mathbf{r}}(\mathbf{r})= & \int_{0}^{\infty}\left[4 \pi \kappa I_{\mathrm{b}}(\mathbf{r})-\int_{V} \mathbf{g}\left(\mathbf{r}, \mathbf{r}_{\mathrm{e}}\right) 4 \pi \kappa I_{\mathrm{b}}\left(\mathbf{r}_{\mathbf{e}}\right) \frac{d \mathbf{r}_{\mathbf{e}}}{d V}\right. \\
& \left.-\int_{A} \mathbf{h}\left(\mathbf{r}, \mathbf{r}_{\mathrm{b}}\right) q_{\mathrm{e}}\left(\mathbf{r}_{\mathrm{b}}\right) \frac{d A_{\mathrm{b}}}{d V}\right] d \lambda
\end{aligned}
$$

The $\mathbf{g}$ and $\mathbf{h}$ tensors are best understood as analogues to view factors used in the enclosure method [20]. The $\mathbf{g}$ tensor contributions account for volumetric absorption based on volumetric emission, while the $\mathbf{h}$ tensor contributions account for volumetric absorption based on emission at the boundary of the computational domain. The advantage of Eq. (6) is recognized when considering that the $\mathbf{g}$ and $\mathbf{h}$ tensors depend on the geometry, boundary conditions, and optical properties of the setup only, and not on the temperature distribution (under the assumption of temperature-independent material properties). The computationally expensive ray tracing is therefore decoupled from the temperature. This implies that $\mathbf{g}$ and $\mathbf{h}$ can be computed once only, and not at each time step. This constitutes a significant gain in computational efficiency. Eq. (6) is reformulated for the discretized domain (Eq. (7)).

$$
\begin{aligned}
\nabla \cdot \mathbf{q}_{\mathrm{r}}(i)= & 4 \pi \kappa \cdot I_{\mathrm{b}}(i)-\sum_{j=1}^{N_{\mathrm{c}}} \mathbf{G}(i, j) \cdot 4 \pi \kappa \cdot I_{\mathrm{b}}(j) \frac{V(j)}{V(i)} \\
& -\sum_{l=1}^{N_{\mathrm{b}}} \mathbf{H}(i, l) \cdot q_{\mathrm{e}}(l) \frac{A_{\mathrm{e}}(l)}{V(i)}
\end{aligned}
$$

For the computation of the temperature field, the divergence of radiative heat fluxes within the computational domain is required. Thus $\mathbf{G}$ and $\mathbf{H}$ must, by definition, have entries for all control volumes absorbing radiative energy. $\mathbf{G}$ and $\mathbf{H}$ contain additional entries accounting for the radiative energy that is absorbed at boundaries. This is required in order to compute the net heat fluxes at the domain boundaries.

\subsection{Monte Carlo ray tracing}

A path length-based Monte Carlo ray tracing method was used for the calculation of the divergence of the radiative heat flux. Monte Carlo approaches are perfectly suited for calculations in complex geometries and are easily and efficiently parallelized. In path length-based Monte Carlo approaches, the starting point of rays is determined stochastically while the absorption is modeled in a deterministic manner. This means that a ray undergoes an exponential decrease in intensity while traveling through a participating medium [22]. We terminated the ray when its energy decreased by 16 orders of magnitude. The limit value is close to the machine precision and therefore has virtually no influence on the results and the conservation of energy. The latter is checked by summing the energy absorbed in any cell or by any boundary face and comparing it to the emitted energy by the control volume or boundary face. We ensured that $100 \%$ of the energy was indeed distributed, with an maximal error of a fraction of $10^{-8}\left(10^{-6} \%\right)$ of the total emitted energy. The ray tracing is fully parallelized and based on an algorithm previously used for particle tracing [23]. Typically the number of rays was in the order of $10^{9}$. The computational time was in the order of a few hours on 16 cores.

The FORTRAN function "RANDOM_NUMBER" was used for generating random numbers together with the GNU Fortran compiler version 4.4.7. The software we developed requires one seed value based on which the number of seed values required by "RANDOM_NUMBER" are obtained from a linear congruential random number generator as described in [24]. The resulting random number generation process was verified in detail, specifically we ensured that for different seed inputs the random numbers were uncorrelated, guaranteeing statistically independent results for different seeds. Additionally it was verified that the random numbers generated on each core are uncorrelated during the parallel execu- 
tion of the software. The ray tracing process is fully separated from the computation of heat fluxes and temperatures.

\subsection{Computational efficiency}

The decoupling of the ray tracing from the temperature field in the computational procedure increases the computational efficiency by several orders of magnitude. The advantage of running the Monte Carlo ray tracing only once is slightly complicated by recognizing that the computational time for the simulation of the temperature distribution scales with $(1-\phi)^{2} N_{\mathrm{c}}^{2}$ if $N_{\mathrm{c}}$ denotes the number of control volumes and $\phi$ the porosity. This is seen from Eq. (7), which constitutes for each control volume $i \in\left[1, N_{\mathrm{c}}\right]$ a sum over all control volumes $j \in\left[1, N_{\mathrm{c}}\right]$. Since the fluid cells are not participating, the higher the porosity, the more efficient the computation.

The computational efficiency can be increased if the radiative heat fluxes are not recomputed at each time step. This idea is naturally given if one realizes that the well-known CFL stability criterion derived from conductive heat transfer imposes small time steps. The temperatures generally change slowly although the temperature gradients could change significantly. Recomputing the divergence of radiative heat fluxes $\left(\nabla \cdot \mathbf{q}_{\mathbf{r}}\right)$, which depends on the temperature distribution, at each time step is not necessary for steady cases. Convergence can be achieved even if the exact computation of $\nabla \cdot \mathbf{q}_{\mathbf{r}}$ at some steps is skipped. We implemented this approach by recomputing $\nabla \cdot \mathbf{q}_{\mathbf{r}}$ only for a fraction of the cells. The cells were chosen based on the temperature difference between present and the last update of $\nabla \cdot \mathbf{q}_{\mathbf{r}}$. The cells, where the temperature has changed the most, distribute their energy based on the new temperatures. To check for convergence, the real residuals must be considered and the exact $\nabla \cdot \mathbf{q}_{\mathbf{r}}$ must be computed. A typical setup was that $20 \%$ of all cells updated their radiative energy distribution each time step and each 100 time steps the exact distribution of $\nabla \cdot \mathbf{q}_{\mathbf{r}}$ was recomputed.

The $\mathbf{G}$ and $\mathbf{H}$ tensors are memory intensive. For $32^{3}$ control volumes they used 6 GB of memory in binary files on the hard drive. Due to the quadratic scaling, doubling the spatial resolution in each direction ( $64^{3}$ control volumes) would require 64 times more memory ( $384 \mathrm{~GB}$ ). The size of the $\mathbf{G}$ and $\mathbf{H}$ tensors can significantly slow the computations if they do not fit into RAM. In that case, the required values must be read from the hard drive whenever needed. It is worth noting that $\mathbf{G}$ and $\mathbf{H}$ are sparse. The zero entries for non-participating cells are not stored. But even in the participating media many entries are close to zero or vanish. It could be envisaged to optimize the memory requirements by ignoring entries below a given threshold. This was not done since no consistent solution for energy conservation could be found. Energy conservation is a crucial requirement for consistency and convergence of any numerical method solving conservation equations.

\subsection{Verification}

The software is verified against analytical and published solutions of problems including radiative and conductive heat transfer treated as separate phenomena, and for problems considering full coupling of both modes.

The computation of the conductive fluxes has been verified by comparing time-dependent temperature profiles with analytical solutions for the problem of two materials with different properties in direct contact [25].

The computation of the radiative fluxes incident and leaving boundaries was verified by considering the setup of two infinitely extended, parallel plates, and was compared with analytical solutions [20] and reference results [26]. The internal emission and absorption was verified by comparison with results from $[27,28]$ considering the case of a quadratic enclosure with black walls of length $L$, at $T=0 \mathrm{~K}$, filled with a grey participating medium at a constant temperature. Fig. 3 shows the comparison of the numerical results obtained with the analytical solution (Appendix A) for the normalized incoming radiative heat flux $\left(\dot{q}_{\text {rad,in }} \cdot\left(n^{2} \sigma_{\mathrm{B}} T^{4}\right)^{-1}\right)$. Eq. (11) in Appendix A was integrated numerically by discretizing both angles $\varphi$ and $\theta$ into 4000 discrete angles. Perfect specular mirrors were used as boundaries perpendicular to the direction of vanishing gradients in order to approximate the two-dimensional setup of the reference problem with the present three dimensional code. $2 \cdot 10^{10}$ rays were used for the Monte Carlo ray tracing for $\kappa L=10$ and a spatial resolution of $81 \times 81,10^{10}$ rays for $\kappa L=1$ and $\kappa L=0.1$ and a spatial resolution of $31 \times 31$. The root mean square deviation of the comparison of the exact solution with our calculations remains below $1.7 \cdot 10^{-3}$ for all cases. This deviation results from the statistical nature of the Monte Carlo ray tracing approach and the spatial discretization of the boundaries. The high number of rays needed to reach that accuracy is due to the fact that the method developed in the present study is computationally inefficient for this verification problem, which is significantly different from the setup considered in this study (Section 4).

The radiation-conduction coupling was verified using results from Viskanta and Grosh [29], considering the case of two infinite, parallel black plates filled with a participating medium. The results in [29] are given for different conduction-toradiation parameters $N=k \cdot \beta /\left(4 \sigma_{B} n^{2} T^{3}\right)(N=0,0.01,0.1)$. Again perfect specular mirrors were used in the directions of vanishing gradients to mimic the one-dimensional setup of the reference problem. $1.28 \cdot 10^{9}$ rays were used for the case of $N=0$, $0.128 \cdot 10^{9}$ rays for the cases of $N=0.01$ and 0.1 for the Monte Carlo ray tracing. A spatial resolution of 81 data points was used. Fig. 4 shows the comparison of our results for the temperature profile, compared to the results obtained in Viskanta and Grosh [29]. The root mean square deviations remain below $2 \cdot 10^{-3}$ for all cases. Some deviation is expected due to the digitization of

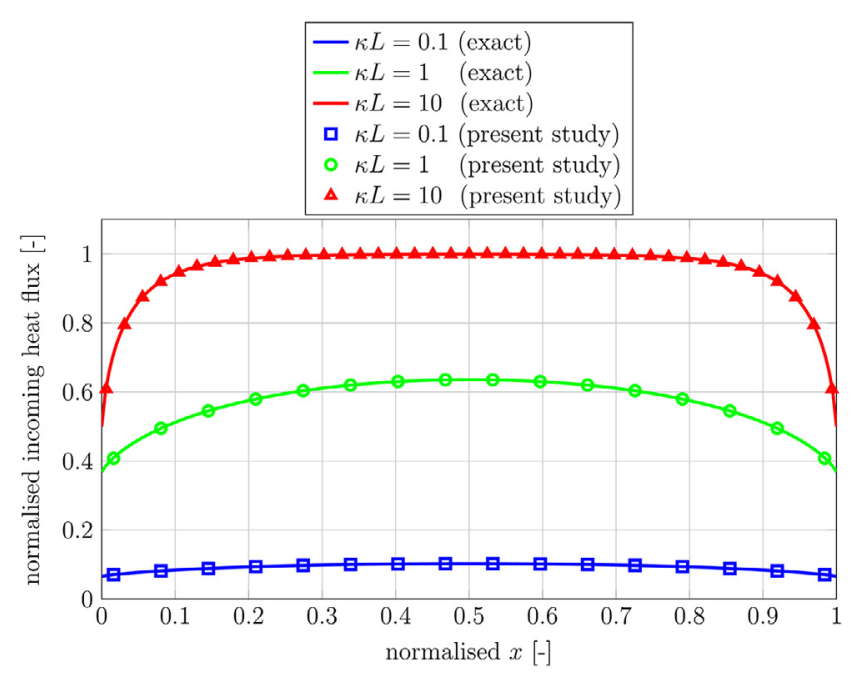

Fig. 3. Comparison of the exact results and our computational results for normalized radiative heat fluxes incident on the walls of a square cavity with black walls at $T=0 \mathrm{~K}$, filled with a grey participating medium at prescribed temperature. RMS deviations for the different cases are $0.23 \cdot 10^{-3}$ for $\kappa L=0.1$, $1.1 \cdot 10^{-3}$ for $\kappa L=1$, and $1.7 \cdot 10^{-3}$ for $\kappa L=10$. 


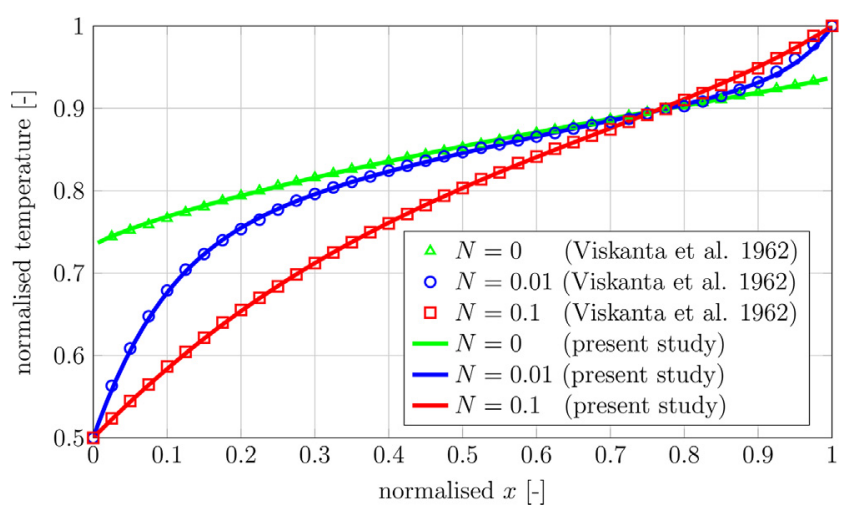

Fig. 4. Comparison of our computational results with the results from Viskanta et al. [29] for the normalized temperature and for different conduction-to-radiation parameters $N$. RMS deviations for the different cases are $0.85 \cdot 10^{-3}$ for $N=0$, $1.9 \cdot 10^{-3}$ for $N=0.01$ and $1.2 \cdot 10^{-3}$ for $N=0.1$.

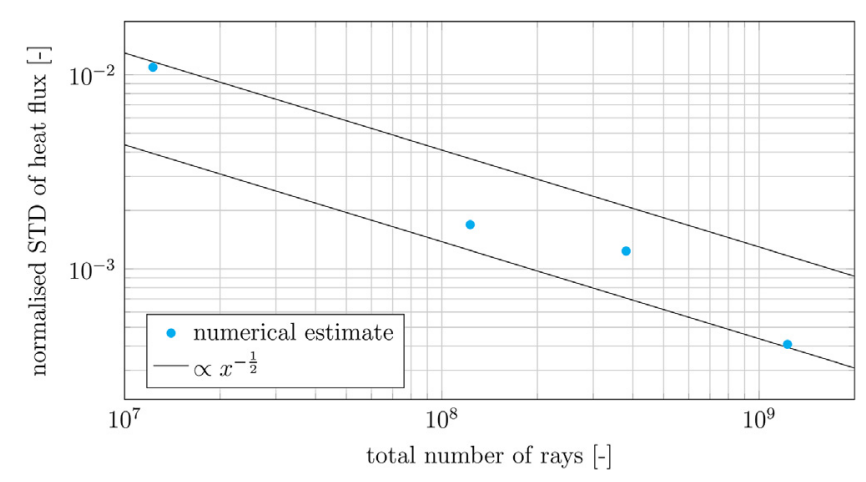

Fig. 5. Standard deviation obtained for the radiative heat fluxes in a radiation only simulation of the baseline geometry estimated from four different realizations. Setup was: $k_{\mathrm{s}}=12, k_{\mathrm{f}}=5, \kappa L=2, n=2$, boundary temperatures were $1650 \mathrm{~K}$ and $1750 \mathrm{~K}$.

the curves from [29] that was carried out with the software "Engauge digitizer".

Fig. 5 shows the estimated standard deviation for the radiative only heat transfer for the geometry and boundary conditions presented in Section 4. Two phases were used, such that the consistent implementation of reflection and refraction at phase boundaries is verified. The thermal properties were $k_{\mathrm{s}}=12, k_{\mathrm{f}}=5$, the optical properties $\kappa L=2, n=2$, and the boundary temperatures were $1650 \mathrm{~K}$ and $1750 \mathrm{~K}$. The optical thickness is given with reference to the sample size (not the pore size). The standard deviation was obtained from four simulations using different seeds for the random number generator. The expected square root decrease of the standard deviation is observed, thus demonstrating the consistency of the Monte Carlo ray tracing approach.

\subsection{Stability and convergence}

For conduction only simulations the stability restrictions are well known and governed by the CFL number. No detailed stability investigations were performed for the radiation only case. In coupled cases it was observed that stability does slightly depend on the optical properties but is similar to the restrictions imposed by the CFL number. This could be explained by the smoothing influence of the radiative heat transfer. The development of instabilities, e.g. hot spots is counteracted by internal radiative emission that removes energy at a fast rate from hot spots. Convergence was assessed based on the 1,2 and infinity norms of the normalized residuals of the temperatures $e=\frac{\left.\frac{\partial T_{i}}{\partial t}\right|_{\text {numerical }}}{T_{i}}$. It was made sure that the 2 norm of $e,\|e\|_{2}=\sqrt{\frac{1}{N_{c}} \sum_{i=1}^{N_{c}} e^{2}}$, did always decrease by at least 6 orders of magnitudes compared to the initial $\|e\|_{2}$ based on the initialization with constant temperatures.

\section{Results}

\subsection{Computational domain}

The geometrical setup chosen for the quantification of the radiation-conduction coupling effects is illustrated in Fig. 6. The solid (participating) phase is shown in solid, the fluid (nonparticipating) phase surrounds it to form a cube. The domain boundaries are indicated. The porosity of the sample is 0.5 . The optical properties of the solid phase were varied, the properties of the fluid phase were fixed at $m_{\mathrm{f}}=1-0 \cdot \mathrm{i}$. As illustrated by the red-blue colors, heat transfer occurs in one direction. The two boundaries perpendicular to the main direction of heat transfer are black plates with prescribed temperatures. The lateral walls are set to be almost perfect specular mirrors to mimic periodic boundary conditions. The reflectivity of the mirrors was set to 0.99999 . This value was chosen in order to avoid infinite reflection of rays perpendicular to the mirrors in the non-participating phase. The maximum radiative heat flux absorbed by the lateral walls for the baseline geometry was $0.2 \%$ of the total transmitted heat flux ( $0.4 \%$ for the case of the inversed geometry).

\subsection{Spatial discretization}

The geometry can be perfectly approximated by a structured mesh. The domain is discretized in $32 \times 32 \times 32$ cubes of same size. The dimension of the cubical domain, $L$, is $1 \mathrm{~cm}$. For the conductive heat fluxes, the mesh must be fine enough to accurately approximate the temperature gradients. For the radiative heat fluxes, the mesh must be fine enough such that radiative heat exchange between different cells can occur. If for a given mesh the optical thickness is increased too much, all rays emitted may be absorbed within the cell of origin. Thus the radiative heat transfer would un-physically disappear. This artificial vanishing of radiative heat transfer limits the optical thickness for a given discretization.

In order to demonstrate that the mesh is well adapted, a refinement study was carried out for the setup of the baseline geometry contained between two black plates at temperatures $1650 \mathrm{~K}$ and $1750 \mathrm{~K}$, with a refraction index of $n=2$ for the participating phase, an optical thickness of $\kappa L=2$ (based on sample size) and a conductivity ratio of $k_{\mathrm{f}} / k_{\mathrm{s}}=0.42$. For conduction only, the evaluated heat fluxes changed less than $0.2 \%$ for a refinement from $32^{3}$ cells to $92^{3}$ cells. The refinement of the mesh for the coupled heat transfer is computationally more expensive. The mesh was refined from $32^{3}$ to $40^{3}$ cells which resulted in a change of the coupled conductive and coupled radiative heat fluxes by $0.01 \%$. Based on these results, it is concluded that the mesh is well adapted. Ideally such a refinement study would be performed for each setup. This would however result in a disproportionate computational effort. The coupling effects that were observed are in the order of several percent and thus two orders of magnitude above the expected numerical uncertainty caused by the spatial discretization.

\subsection{Statistical uncertainty}

The Monte Carlo ray tracing introduces an uncertainty due to the statistical nature of the approach. This uncertainty is com- 

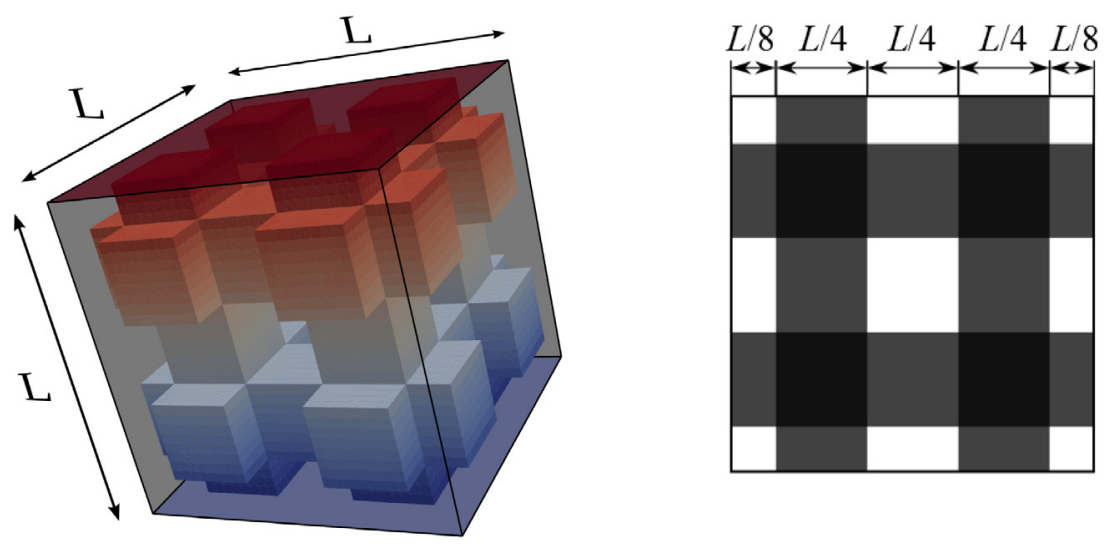

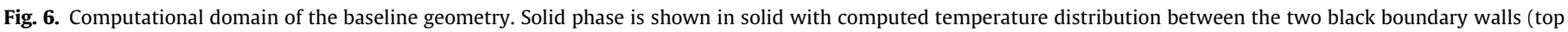
and bottom) at prescribed temperature. The fluid phase is complementing the solid phase to form a cube. Left: three dimensional view, right: side/top projection.

monly quantified by the standard deviation that is expected for a given number of rays. In the present setup internal emission and emission from boundaries is considered, resulting in the $\mathbf{G}$ and $\mathbf{H}$ tensors. The computation of those tensors is decoupled from the temperatures. Without knowledge of the temperature distribution it is not possible to assign an energy to a ray. Therefore the number of rays emitted from each cell and boundary face is equal. A uniform distribution of energy per ray would reduce the statistical uncertainty, however this is not feasible due to the implicit nature of the problem. The standard deviation of the heat fluxes can be estimated by running the same simulation several times using a different set of random numbers. In computational terms the random number generator is seeded differently. This was done for the same setup as the spatial refinement study (Section 4.2). $6 \cdot 10^{4}$ rays were used per cell and $1.2 \cdot 10^{5}$ rays per boundary face resulting in a total number of $1.12 \cdot 10^{9}$ rays. The standard deviation obtained for the radiative heat fluxes in a radiation only simulation based on 4 different runs is $0.05 \%$. This uncertainty is insignificant compared to the coupling effects that are presented and it is concluded that it is appropriate to use the proposed number of rays.

\subsection{Non-dimensionalization of results}

The parameter space to be investigated is defined as the optical and thermal material properties, morphological characteristics, and the boundary temperatures. Different regimes exist: (i) the regime where conductive heat transfer dominates (e.g. at low temperatures), and (ii) the regime where radiative heat transfer dominates (e.g. at high temperatures). This is commonly quantified in the "conduction-to-radiation parameter" defined as $N=k \kappa /\left(4 \sigma_{B} n^{2} T^{3}\right)$. Özisik gives a comprehensive introduction to non-dimensional quantities connected to coupled conductiveradiative heat transfer in [30]. This conduction-to-radiation parameter is problematic for two reasons: first it is unclear what temperature to use if a range of temperatures is observed, and second it is far from unity when conduction and radiation are equivalent. The importance of the relation between conductive and radiative heat transfer however remains unquestioned. Here we show the results with respect to $\xi=\frac{\left.\dot{q}_{\text {rad }}\right|_{\text {coupled }}}{\left.\dot{q}_{\text {cond }}\right|_{\text {coupled }}+\left.\dot{q}_{\text {rad }}\right|_{\text {coupled }}}$, expressing the fraction of the heat transferred that is occurring through radiation. The choice of $\xi$ is based on the observation that this nondimensionalization significantly reduces the parameter space and on the physical insight it provides. The coupling effect, $\zeta$, is defined as the ratio of the total heat transferred in the case of coupled conductive and radiative heat transfer to the superposition of the con- ductive and radiative heat transfer obtained from simulations considering radiation only and conduction only

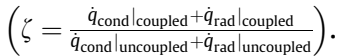

\subsection{Reduction of parameters through non-dimensionalization}

The goal of the study is to summarize the results in a concise way. The non-dimensionalization proposed in Section 4.4 reduces the parameter space. Results for different material properties and boundary conditions are shown in Fig. 7. For a given physical setup, the boundary conditions were varied to obtain data points at different $\xi$ for that specific setup. The optical properties of the solid phase and the thermal properties of both phases are given in the legend. The optical thickness is calculated with respect to the sample size $L$. The temperature boundary conditions were chosen realistically, the maximum temperature $T_{\max }$ and the value of $\left(T_{\max }-T_{\min }\right) / T_{\max }$ are indicated in the plot for each point. We observed that the data points obtained for setups of same optical properties and same ratios of conductivities $\left(k_{\mathrm{f}} / k_{\mathrm{s}}\right)$ align on one single curve, independently of the exact values of conductivity, boundary temperatures, and difference of boundary temperatures. This result justifies the non-dimensionalization (use of $\xi$ ) introduced in this work. For the sake of readability, the temperature levels are omitted in all figures except 7 , but are given in Appendix B.

The extreme values observed and plotted in Fig. 7 are readily explained. The case of $\xi=0$ corresponds to conduction only, $\xi=1$ to radiation only. Naturally there is no coupling effect if one single mode of heat transfer is present, thus the $y$-value is unity. For values $0<\xi<1$ a clear coupling effect is observed, enhancing the total heat transfer. A discussion of why an enhancement is observed is given in Section 5. It could be expected that maximal $\zeta$ occurs at $\xi=0.5$ where conduction and radiation are equivalent. However, since coupling occurs in the participating phase, where the divergence of radiative fluxes does not vanish (Eq. (1)), the maximum in $\zeta$ is not necessarily at $\xi=0.5$. Equivalence of radiative and conductive fluxes for the full cross section does not mean that both modes are transferring an equal amount of heat in the participating phase. This explains the different locations of the maximum $\zeta$. The strength of the coupling depends on the setup and is discussed below. The radiative heat fluxes across the sample are evaluated at the top and bottom boundaries. In the cases investigated in the present study the heat fluxes were approximately constant along the main direction of heat transfer (i.e. within $3 \%$ for $\Delta T=100 \mathrm{~K}$ ). The average between inlet and outlet was used. 


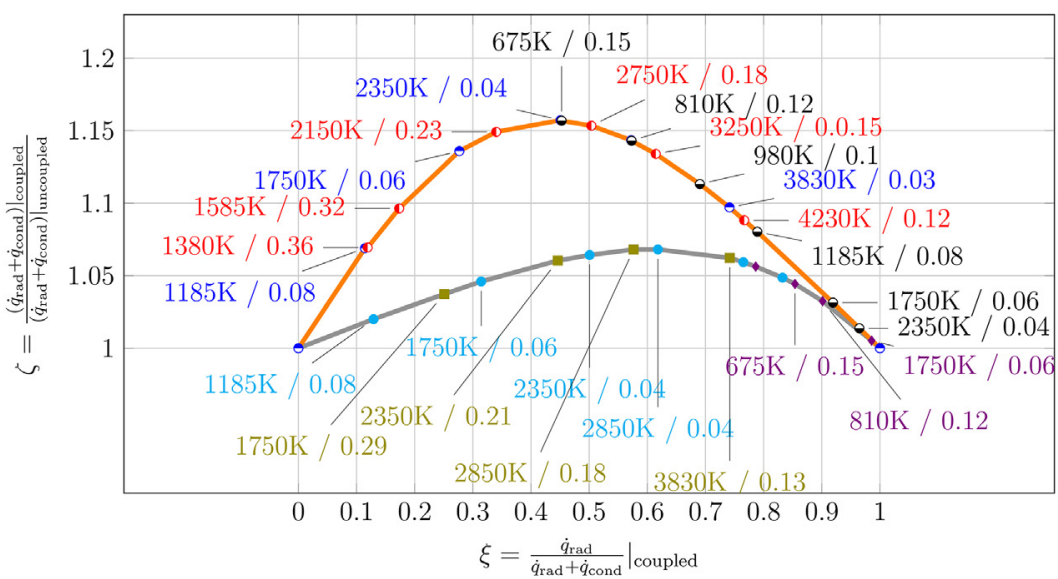

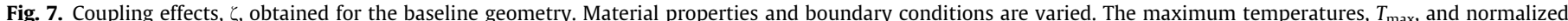
differences between maximum and minimum temperatures, $\left(T_{\max }-T_{\min }\right) / T_{\max }$, are indicated.
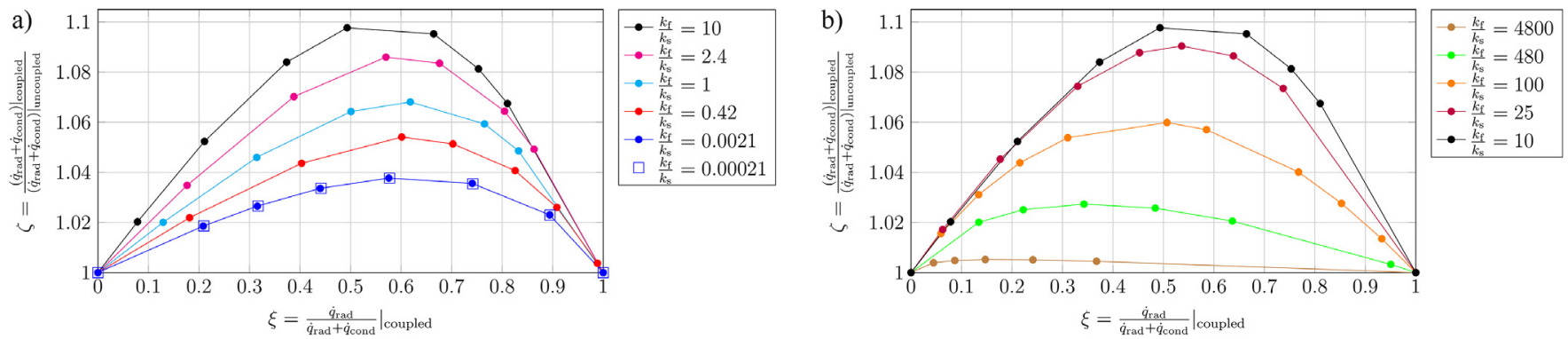

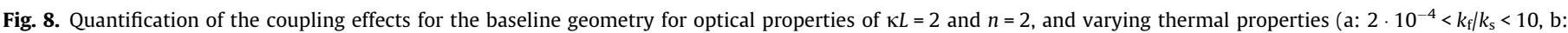
$\left.10<k_{\mathrm{f}} / k_{\mathrm{s}}<4800\right)$.

\subsection{Parameter variation: thermal properties}

Results show that the conductivities, or more precisely the conductivity ratio $k_{\mathrm{f}} / k_{\mathrm{s}}$, are key parameters. Fig. 8 show the coupling effects obtained for $\kappa L=2, n=2$, and varying conductivities. The coupling effects vanish for very large conductivity ratios. This can be explained since in the limit of $k_{\mathrm{f}} / k_{\mathrm{s}} \rightarrow \infty$ conduction can only occur in the fluid phase, while radiation occurs only in the solid phase. Interaction between the two modes is limited to the interfaces and becomes negligible. A similar case where the interaction is limited to the interface, namely the situation of an opaque phase and a transparent phase was investigated in [31] which concluded that no coupling effects exist in that case.

In the case of $k_{\mathrm{f}} / k_{\mathrm{s}} \rightarrow 0$ conduction and radiation occur in the solid phase only. For very low conductivity ratios, the coupling effect converges to a distinct curve (Fig. 8a). Further reduction in the fluid phase conductivity (below $k_{\mathrm{f}} / k_{\mathrm{s}}=2 \cdot 10^{-3}$ ) does not change $\zeta$. The extreme case of the fluid phase not participating in the heat transfer is reached.

Fig. 9 shows the maximum coupling effect observed for any $\xi$ as a function of the conductivity ratios for different optical thicknesses at $n=2$. Increasing the optical thickness increases the coupling. This is expected since increasing optical thickness means increasing the internal emission and absorption, increasing the amount of radiative energy exchanged within the participating phase, thus creating stronger interactions. In this case the influence of the conductivity ratio is more important. A distinct peak is observed around a conductivity ratio of $k_{\mathrm{f}} / k_{\mathrm{s}}=10$, for all optical thicknesses. We expect that the conductivity ratio at the peak $\zeta$ will change for different geometries while occurring at a similar conductivity ratio for all three optical thicknesses.

Fig. 10 shows the $\xi$ at which the maximum $\zeta$ is observed for different conductivity ratios and refractive indices at an optical thickness of $\kappa L=2$. For very low conductivity ratios $k_{\mathrm{f}} / k_{\mathrm{s}}$, the location of the coupling converges to a certain value, corresponding to the situation of heat transfer occurring only in the solid phase. The value is depending on $n$, i.e. for different indices of refraction $\xi$ for maximum $\zeta$ are different. The internally emitted radiation $\left(n^{2} \sigma T^{4}\right)$ increases with $n$. Therefore in order to reach optimal coupling for larger $n$, $\xi$ must be chosen such that the fraction of heat transferred through conduction increases and can compensate the increase in internal radiative emission. Thus when increasing $n$ the location for maximum coupling moves to lower $\xi$. Increasing the conductivity ratio $k_{\mathrm{f}} / k_{\mathrm{s}}$ moves the location of maximum coupling towards lower $\xi$ values. An increasing conductivity ratio weakens the conductive heat transfer in the solid phase where the coupling occurs. Radiation must be weakened in order to compensate for the weaker conduction and to achieve optimal coupling. It is expected that for higher conductivity ratios the location of maximum coupling approaches $\xi=0$. This approach is accompanied by the disappearing of the coupling effect and consistent with the previous statement that the coupling effects vanish at $\xi=0$.

\subsection{Parameter variation: optical properties}

Figs. 11 and 12 quantify the maximum coupling effect observed for any $\xi$ for the baseline geometry with a conductivity ratio of $k_{\mathrm{f}} / k_{\mathrm{s}}=1$ and varying optical properties. 


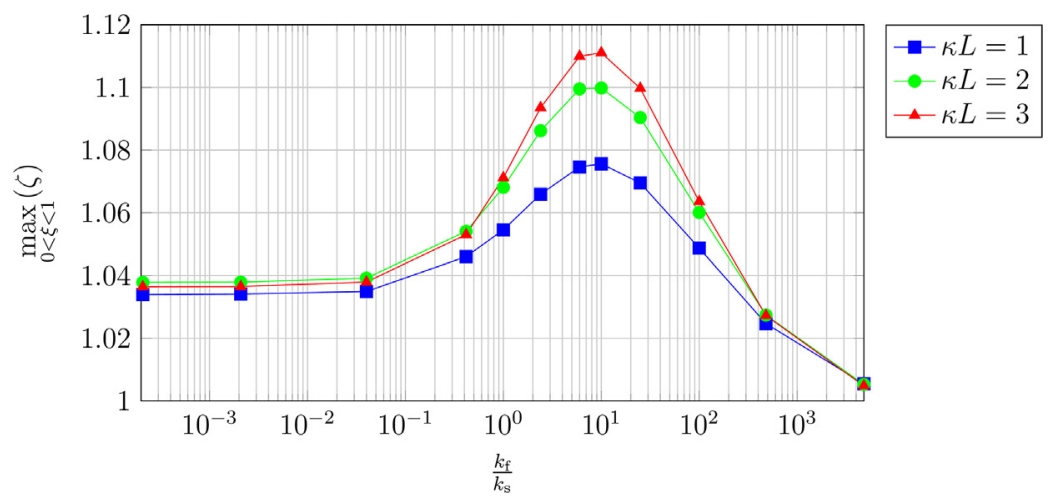

Fig. 9. Maximum coupling effect observed for any $\xi$ for the baseline geometry at $n=2$, for different optical thicknesses and varying conductivity ratios.

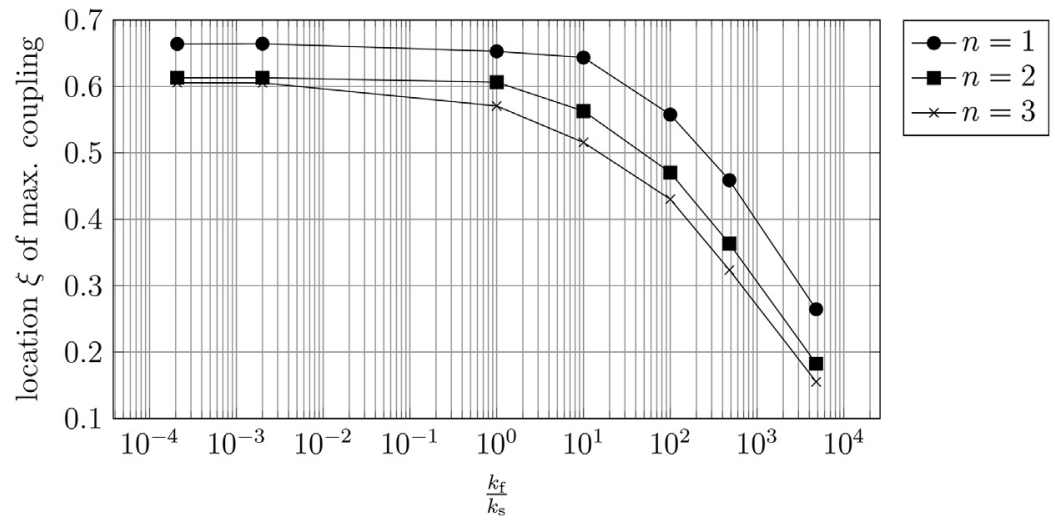

Fig. 10. $\xi$ at which maximum $\zeta$ is observed for the baseline geometry and optical thickness of $\kappa L=2$, for varying refraction indices and conductivity ratios.

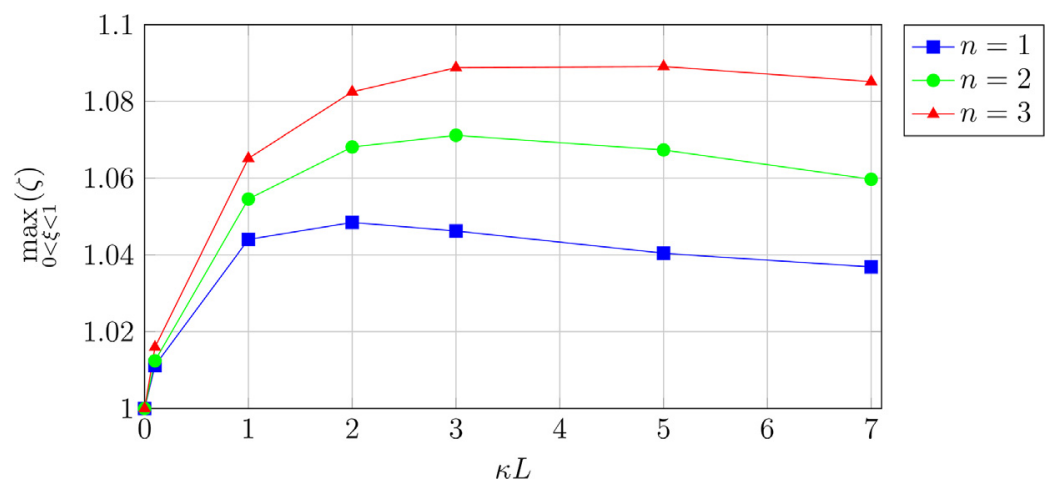

Fig. 11. Maximum coupling effect observed for any $\xi$ for the baseline geometry, conductivity ratio of $k_{\mathrm{f}} / k_{\mathrm{s}}=1$, and varying indices of refraction and optical thickness.

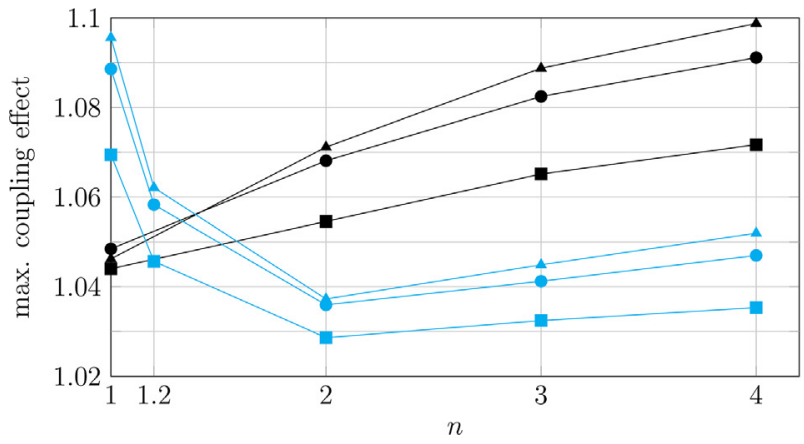

- baseline geometry $\kappa L=1$

- baseline geometry $\kappa L=2$

$\sim$ baseline geometry $\kappa L=3$

- inverted geometry $\kappa L=1$

- inverted geometry $\kappa L=2$

$\sim$ inverted geometry $\kappa L=3$

Fig. 12. Maximum coupling effect observed for any $\xi$ for the baseline and inverted geometry, conductivity ratio of $k_{\mathrm{f}} / k_{\mathrm{s}}=1$, and varying optical thicknesses and $n$. 
Fig. 11 shows that at an optical thickness of zero the coupling effect vanishes as no radiative emission or absorption occurs. The divergence of radiative heat fluxes vanishes and both modes of heat transfer are decoupled. Increasing the optical thickness increases the coupling effects. After a steep initial increase the maximum coupling effects stabilize before decreasing. A decrease for large optical thicknesses is expected as a case similar to the setup of an opaque and a transparent phase is approached. In that case the interaction will be limited to the interfaces. The vanishing of the coupling effects is also confirmed by [31]. Results for higher optical thicknesses could only be obtained by using a refined mesh according to the logic outlined in Section 4.2.

Fig. 12 shows results obtained for the baseline geometry and for a geometry where the solid and fluid phases were switched with reference to Fig. 6 . For the baseline geometry, the coupling effect increases with increasing $n$ attributed to two physical effects. First the emission from black boundaries into a participating medium and the emission from a participating medium is proportional to $n^{2}$. And second the reflection and refraction behavior at phase interfaces changes with varying $n$. For the normal geometry, the trend is clear, increasing $n$ increases the coupling effect. This is expected since the reflection and refraction at the phase boundaries change with increasing $n$ such that more radiative energy is reflected when going from the solid to the fluid phase. This avoids that radiative energy leaves the participating phase and allows for increased coupling. Discussion of the results for the "inverted geometry" is deferred to Section 4.8. Results for larger $n$ are not shown since materials with $n>4$ are of less relevance in the engineering context.

\subsection{Parameter variation: geometry}

Fig. 12 shows the results for simulations performed with the same material properties and boundary conditions for the baseline geometry (Fig. 6) and for the inverted geometry. In the case of the inverted geometry, an initial decrease of the coupling effect is observed, before the coupling effect increases again as $n$ is increased. This trend is different than that observed for the baseline geometry and is believed to be caused by boundary effects. For the baseline geometry, $25 \%$ of the surface adjacent to the black boundaries was covered by the solid phase. For the inverted geometry this changes to $75 \%$. A clarification can be obtained by considering two extreme cases: Parallel and serial slabs. These two cases constitute extreme cases of porous geometries and various effects are expected to be dominating or suppressed. Two setups exist for serial and parallel slabs as shown in Fig. 13b. Due to the periodic boundary conditions on the lateral walls, results for baseline and inverted parallel slabs coincide and the distinction between baseline and inverse parallel slabs is not made in Fig. 13a. The results for serial slabs are significantly different depending on which phase is adjacent to the boundaries perpendicular to the main direction of heat transfer. This is shown in Fig. 13a for the case of $k_{\mathrm{f}} / k_{\mathrm{s}}=1, \kappa L=2, n=2$. It is expected that the coupling for the baseline and inverted geometry are bounded by the coupling for parallel and serial slabs. It becomes apparent, that the baseline and inverted serial slabs constitute the two extreme cases. The coupling for the parallel slabs is between the two cases of baseline serial and inverted serial slabs. The stronger coupling effects for the baseline serial slabs can be explained. In the case of the inverted serial slabs, much of the radiation internally emitted in the participating slabs adjacent to top and bottom boundaries is absorbed by the black boundaries and does not contribute much to the interaction. The same effect is believed to keep the coupling of the inverted geometry below that of the baseline geometry in Fig. 12.

\section{Application of results to engineering problems}

The results clearly indicate that a correction is necessary when superimposing existing models for conduction and radiation in order to obtain accurate heat transfer predictions in porous media. Therefore the results provide practical relevance in engineering applications, in addition to its scientific merit. The reduction of complexity that can be achieved combining and adapting existing models is illustrated by showing the temperature profile obtained for the case of the baseline geometry for $\kappa L=3, n=2, k_{\mathrm{f}} / k_{\mathrm{s}}=10$, between black boundaries at temperatures of $2750 \mathrm{~K}$ and $2850 \mathrm{~K}$. Fig. 14 shows the temperature distribution at different locations on the axis in direction of main heat transfer, $z$. The temperature was averaged at all discrete values $z_{i}$ for all cells (of both, fluid and solid phase) with cell center at $z_{i}$.

The temperature profile for radiative heat transfer only exhibits the well-known temperature slip at the walls. This discontinuity in temperature shows that this is not a realistic case. Even in materials with very low conductivity, the heat conduction will force the discontinuity to disappear. This is observed for the temperature distribution of the coupled case. These three temperature profiles allow a prediction of the coupling effects. The temperature profile for conduction only changes when it is coupled with radiation such that gradients at the boundaries become steeper. Thus it is expected that the conductive heat transfer is enhanced. The temperature profile for radiation only changes such that the cold and warm temperatures close to the boundaries become even colder and warmer, respectively. Considering that the boundaries are black, this will decrease the net heat transfer between the a)

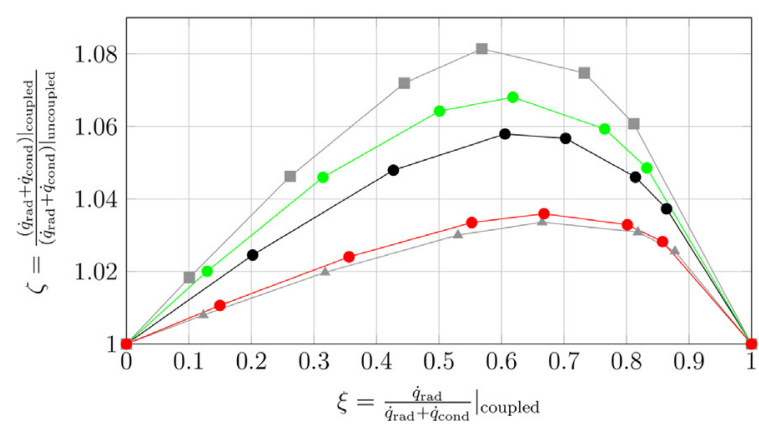

b)

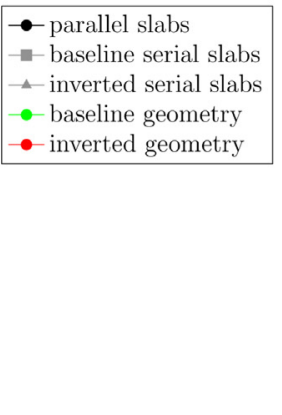

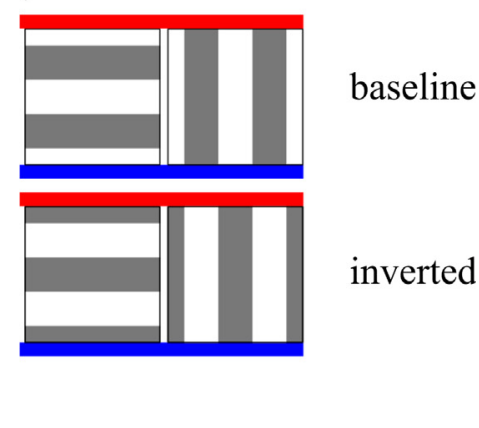

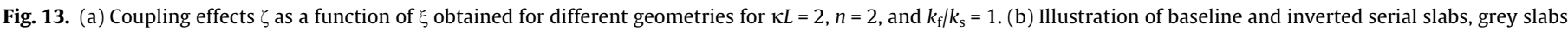
illustrate the solid phase (participating) and white slabs illustrate the fluid phase (non-participating). 


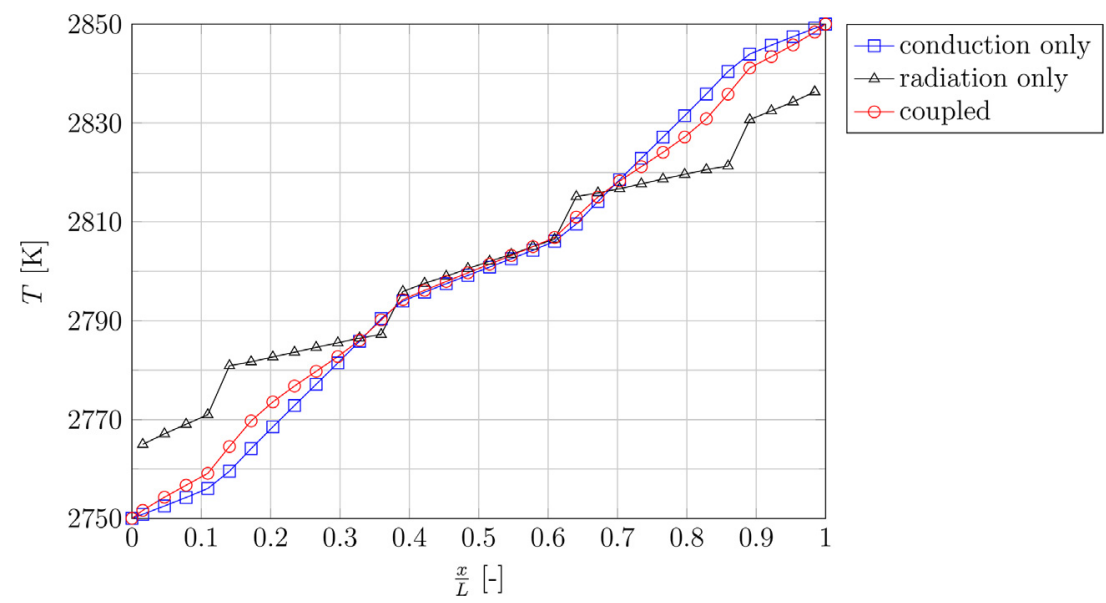

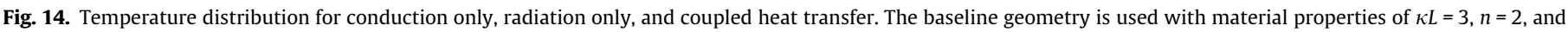

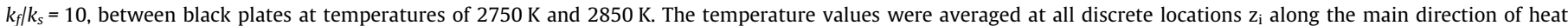
transfer.

boundaries, thus the radiative heat transfer is expected to decrease. Indeed in this case, the conductive heat transfer increased by a factor of 1.47 and the radiative heat transfer decreased by a factor of 0.85 . The total amount of heat transferred increased by $11 \%$.

The understanding of the influence of coupling of radiative and conductive heat transfer allows for an immense simplification when calculating coupled radiation-conduction engineering problems. The resolution of the pore-scale geometry is avoided when utilizing the volume averaging theory. The knowledge of the coupling effect allows neglecting the coupling terms and instead weighting radiation and conduction heat transfer to obtain an accurate heat transfer prediction. This procedure allows for the proper use of existing and validated effective properties, treating conduction and radiation independently and adding a correction that accounts for coupling effects.

The present study provides a first step towards the computation of coupled radiation-conduction heat transfer in complex geometries. Important challenges remaining include the further investigation of the influence of porosity, different complex morphologies and the simulation of larger, samples with higher optical thicknesses to discuss the influence of the boundaries on the coupling.

\section{Conclusion}

A numerical scheme based on Monte Carlo ray tracing and finite volumes has been developed and used to solve the coupled radiative-conductive heat transfer for domains discretized in a structured mesh. The computational efficiency was significantly improved to allow running numerous simulations to investigate the influence of various parameters on the strength of the coupling effect.

A modified conduction-to-radiation parameter was introduced allowing to quantify the coupling effect independently of the boundary temperatures but based on the relative strength of the radiative and conductive heat transfer. It was shown that in terms of thermal properties in the steady case, the ratio of conductivity between the two bulk phases is governing the coupling effect.

A full discussion is presented, highlighting the dependency of the coupling effect on thermal and optical properties. It was found that the influence of coupling can be significant, up to $15 \%$. However for a given setup with bulk heat transfer properties, the maximum coupling is reached for a given set of boundary conditions only.

Increasing optical thickness increases the coupling up to a peak, after which increasing the optical thickness decreases the coupling. A distinct peak was observed occurring at a specific conductivity ratio. It was shown that the effect of the index of refraction can be different for different geometries. This is due to boundary effects and demonstrated by showing results for the setup of serial slabs.

Further optimizations of the computational method can allow to simulate realistic geometries obtained from tomography. This will lead to valuable quantitative results applicable to realistic materials thus improving engineering calculations.

\section{Acknowledgement}

This material is based upon work performed in cooperation with CTI Swiss Competence Centers for Energy Research (SCCER Heat and Electricity Storage). We thank Selmar Binder and Jeremy Mora-Monteros for fruitful discussions, Vincent Leroy for insightful comments, and Fouad Aabid for the initial efforts.

\section{Appendix A. Analytical solution for setup from $[27,28]$}

$$
\begin{aligned}
& x \in[0,1] \\
& \theta_{1}=\operatorname{atan}\left(\frac{1 / \cos (\varphi)}{1-x}\right) \\
& \theta_{2}=\pi-\operatorname{atan}\left(\frac{1 / \cos (\varphi)}{x}\right) \\
& \frac{\dot{q}_{\text {rad, in }}(x)}{n^{2} \sigma T^{4}}=2 \cdot \int_{\varphi=0}^{\pi / 2}\left[\begin{array}{c}
\int_{\theta=0}^{\theta=\theta_{1}}\left\{1-\exp \left(-\frac{1-x}{\cos (\theta)}\right)\right\} \cdot \sin ^{2}(\theta) d \theta+ \\
\theta=\theta_{1} \\
\int_{\theta=\theta_{2}}^{\pi}\left\{1-\exp \left(-\frac{1 / \cos (\theta)}{\sin (\theta)}\right)\right\} \cdot \sin ^{2}(\theta) d \theta+
\end{array}\right] \cdot \cos (\varphi) d \varphi
\end{aligned}
$$


Appendix B. Temperature levels and coupling effects for all data points presented

\begin{tabular}{|c|c|c|c|c|c|c|c|}
\hline$\kappa L[-]$ & $n[-]$ & $k_{\mathrm{f}}[\mathrm{W} / \mathrm{m} / \mathrm{K}]$ & $k_{\mathrm{s}}[\mathrm{W} / \mathrm{m} / \mathrm{K}]$ & $T_{\max }[\mathrm{K}]$ & $T_{\min }[\mathrm{K}]$ & $\xi[-]$ & $\zeta[-]$ \\
\hline 2 & 2 & 12 & 12 & 4600 & 4500 & 0.832504 & 1.0485746 \\
\hline 2 & 2 & 12 & 12 & 3830 & 3730 & 0.7650841 & 1.0592955 \\
\hline 2 & 2 & 12 & 12 & 2850 & 2750 & 0.6182344 & 1.0680754 \\
\hline 2 & 2 & 12 & 12 & 1750 & 1650 & 0.3143344 & 1.0459769 \\
\hline 2 & 2 & 12 & 12 & 1185 & 1085 & 0.1291709 & 1.0200638 \\
\hline 2 & 2 & 12 & 12 & 1750 & 1250 & 0.2509385 & 1.0372842 \\
\hline 2 & 2 & 12 & 12 & 3830 & 3330 & 0.7418514 & 1.0621817 \\
\hline 2 & 2 & 0.025 & 0.025 & 3830 & 3730 & 0.998555 & 1.0005321 \\
\hline 2 & 2 & 0.025 & 0.025 & 1750 & 1650 & 0.9856476 & 1.0052428 \\
\hline 2 & 2 & 0.025 & 0.025 & 810 & 710 & 0.9014613 & 1.0324124 \\
\hline 2 & 2 & 0.025 & 0.025 & 675 & 575 & 0.8539421 & 1.0442682 \\
\hline 2 & 2 & 50 & 5 & 2350 & 2250 & 0.3735079 & 1.0839455 \\
\hline 2 & 2 & 50 & 5 & 1750 & 1650 & 0.2109367 & 1.05234 \\
\hline 2 & 2 & 50 & 5 & 1185 & 1085 & 0.0783726 & 1.0202701 \\
\hline 2 & 2 & 75 & 3 & 4600 & 4500 & 0.7373368 & 1.0734836 \\
\hline 2 & 2 & 75 & 3 & 3830 & 3730 & 0.6386569 & 1.0864005 \\
\hline 2 & 2 & 75 & 3 & 2850 & 2750 & 0.4526992 & 1.0877339 \\
\hline 2 & 2 & 75 & 3 & 2350 & 2250 & 0.3302216 & 1.0743348 \\
\hline 2 & 2 & 75 & 3 & 1750 & 1650 & 0.1766161 & 1.0452595 \\
\hline 2 & 2 & 75 & 3 & 1185 & 1085 & 0.0627086 & 1.0171769 \\
\hline 2 & 2 & 300 & 3 & 2850 & 2750 & 0.2156683 & 1.0438381 \\
\hline 2 & 2 & 300 & 3 & 2350 & 2250 & 0.1338404 & 1.0311053 \\
\hline 2 & 2 & 12 & 0.025 & 1440 & 1340 & 0.4838818 & 1.0257118 \\
\hline 2 & 2 & 12 & 0.025 & 1185 & 1085 & 0.3425964 & 1.0273441 \\
\hline 2 & 2 & 12 & 0.025 & 980 & 880 & 0.222282 & 1.025097 \\
\hline 2 & 2 & 12 & 0.025 & 810 & 710 & 0.1342503 & 1.0200976 \\
\hline 2 & 2 & 12 & 0.0025 & 1185 & 1085 & 0.3675401 & 1.0045181 \\
\hline 2 & 2 & 12 & 0.0025 & 980 & 880 & 0.241433 & 1.0051149 \\
\hline 2 & 2 & 12 & 0.0025 & 810 & 710 & 0.1470923 & 1.0052207 \\
\hline 2 & 2 & 12 & 0.0025 & 675 & 575 & 0.0867329 & 1.004838 \\
\hline 2 & 2 & 12 & 5 & 4600 & 4500 & 0.8633045 & 1.0492081 \\
\hline 2 & 2 & 12 & 5 & 3830 & 3730 & 0.8047554 & 1.064371 \\
\hline 2 & 2 & 12 & 5 & 2850 & 2750 & 0.6761113 & 1.0835051 \\
\hline 2 & 2 & 12 & 5 & 2350 & 2250 & 0.5700256 & 1.0859624 \\
\hline 2 & 2 & 12 & 5 & 1750 & 1650 & 0.3878588 & 1.0701837 \\
\hline 2 & 2 & 12 & 5 & 1185 & 1085 & 0.1764899 & 1.0348197 \\
\hline 2 & 2 & 5 & 12 & 3830 & 3730 & 0.8257013 & 1.0406758 \\
\hline 2 & 2 & 5 & 12 & 2850 & 2750 & 0.7025128 & 1.0513492 \\
\hline 2 & 2 & 5 & 12 & 1750 & 1650 & 0.4031699 & 1.0436258 \\
\hline 2 & 2 & 5 & 12 & 1185 & 1085 & 0.1814359 & 1.0218977 \\
\hline 2 & 2 & 0.05 & 0.12 & 2850 & 2750 & 0.9885688 & 1.0037539 \\
\hline 2 & 2 & 0.05 & 0.12 & 1185 & 1085 & 0.9083649 & 1.0260115 \\
\hline 2 & 2 & 0.025 & 12 & 3830 & 3730 & 0.8941133 & 1.0230198 \\
\hline 2 & 2 & 0.025 & 12 & 2350 & 2250 & 0.7409072 & 1.0355612 \\
\hline
\end{tabular}


Temperature levels and coupling effects for all data points presented (continued)

\begin{tabular}{|c|c|c|c|c|c|c|c|}
\hline$\kappa L[-]$ & $n[-]$ & $k_{\mathrm{f}}[\mathrm{W} / \mathrm{m} / \mathrm{K}]$ & $k_{\mathrm{s}}[\mathrm{W} / \mathrm{m} / \mathrm{K}]$ & $T_{\max }[\mathrm{K}]$ & $T_{\min }[\mathrm{K}]$ & $\xi[-]$ & $\zeta[-]$ \\
\hline 2 & 2 & 0.025 & 12 & 1750 & 1650 & 0.5756989 & 1.0377133 \\
\hline 2 & 2 & 0.025 & 12 & 1430 & 1330 & 0.4399823 & 1.033612 \\
\hline 2 & 2 & 0.025 & 12 & 1185 & 1085 & 0.3155641 & 1.0265187 \\
\hline 2 & 2 & 0.025 & 12 & 980 & 880 & 0.208357 & 1.0185605 \\
\hline 2 & 2 & 0.0025 & 12 & 3830 & 3730 & 0.8945141 & 1.0229419 \\
\hline 2 & 2 & 0.0025 & 12 & 2350 & 2250 & 0.7420014 & 1.0354556 \\
\hline 2 & 2 & 0.0025 & 12 & 1750 & 1650 & 0.5772086 & 1.0376693 \\
\hline 2 & 2 & 0.0025 & 12 & 1430 & 1330 & 0.4415649 & 1.0336303 \\
\hline 2 & 2 & 0.0025 & 12 & 1185 & 1085 & 0.3169943 & 1.0265748 \\
\hline 2 & 2 & 0.0025 & 12 & 980 & 880 & 0.2094765 & 1.0186242 \\
\hline 3 & 4 & 50 & 5 & 3830 & 3730 & 0.7413339 & 1.0971032 \\
\hline 3 & 4 & 50 & 5 & 2850 & 2750 & 0.5722501 & 1.1433664 \\
\hline 3 & 4 & 50 & 5 & 2350 & 2250 & 0.4508065 & 1.1571868 \\
\hline 3 & 4 & 50 & 5 & 1750 & 1650 & 0.2767968 & 1.1358546 \\
\hline 3 & 4 & 50 & 5 & 1185 & 1085 & 0.1145154 & 1.0689616 \\
\hline 3 & 4 & 50 & 5 & 4230 & 3730 & 0.7665835 & 1.0882097 \\
\hline 3 & 4 & 50 & 5 & 3250 & 2750 & 0.6144877 & 1.1339179 \\
\hline 3 & 4 & 50 & 5 & 2750 & 2250 & 0.503702 & 1.15338 \\
\hline 3 & 4 & 50 & 5 & 2150 & 1650 & 0.340168 & 1.1491193 \\
\hline 3 & 4 & 50 & 5 & 1585 & 1085 & 0.1732868 & 1.096392 \\
\hline 3 & 4 & 50 & 5 & 1380 & 880 & 0.1190222 & 1.0694064 \\
\hline 3 & 4 & 1 & 0.1 & 2350 & 2250 & 0.9646362 & 1.0137723 \\
\hline 3 & 4 & 1 & 0.1 & 1750 & 1650 & 0.9192868 & 1.0313953 \\
\hline 3 & 4 & 1 & 0.1 & 1185 & 1085 & 0.7891891 & 1.0802786 \\
\hline 3 & 4 & 1 & 0.1 & 980 & 880 & 0.6903296 & 1.1132289 \\
\hline 3 & 4 & 1 & 0.1 & 810 & 710 & 0.5729138 & 1.1429596 \\
\hline 3 & 4 & 1 & 0.1 & 675 & 575 & 0.4526137 & 1.1567978 \\
\hline
\end{tabular}

\section{References}

[1] S. Whitaker, The Method of Volume Averaging, Springer, Netherlands, 1999.

[2] R. Coquard, D. Rochais, D. Baillis, Experimental investigations of the coupled conductive and radiative heat transfer in metallic/ceramic foams, Int. J. Heat Mass Transf. 52 (2009) 4907-4918, http://dx.doi.org/10.1016/j. ijheatmasstransfer.2009.05.015.

[3] L.K. Matthews, R. Viskanta, F.P. Incropera, Development of inverse methods for determining thermophysical and radiative properties of high-temperature fibrous materials, Int. J. Heat Mass Transf. 27 (1984) 487-495, http://dx.doi. org/10.1016/0017-9310(84)90022-X.

[4] S. Haussener, P. Coray, W. Lipiński, P. Wyss, A. Steinfeld, Tomography-based heat and mass transfer characterization of reticulate porous ceramics for hightemperature processing, J. Heat Transf. 132 (2010) 23305, http://dx.doi.org/ 10.1115/1.4000226.

[5] J. Petrasch, P. Wyss, A. Steinfeld, Tomography-based Monte Carlo determination of radiative properties of reticulate porous ceramics, J. Quant. Spectrosc. Radiat. Transf. 105 (2007) 180-197, http://dx.doi.org/10.1016/j. jqsit.2006.11.002.

[6] M. Tancrez, J. Taine, Direct identification of absorption and scattering coefficients and phase function of a porous medium by a Monte Carlo technique, Int. J. Heat Mass Transf. 47 (2004) 373-383, http://dx.doi.org/ 10.1016/S0017-9310(03)00146-7.

[7] B. Zeghondy, E. Iacona, J. Taine, Determination of the anisotropic radiative properties of a porous material by radiative distribution function identification (RDFI), Int. J. Heat Mass Transf. 49 (2006) 2810-2819, http://dx.doi.org/ 10.1016/j.ijheatmasstransfer.2006.02.034.

[8] B. Zeghondy, E. Iacona, J. Taine, Experimental and RDFI calculated radiative properties of a mullite foam, Int. J. Heat Mass Transf. 49 (2006) 3702-3707, http://dx.doi.org/10.1016/j.ijheatmasstransfer.2006.02.036.

[9] M. Lazard, S. André, D. Maillet, Thermal characterization of semi-transparent media: measurement of phononic diffusivity of glass and silica, Eur. Phys. J. Appl. Phys. 23 (2003) 207-211, http://dx.doi.org/10.1051/epjap:2003020.

[10] M. Lazard, S. André, D. Maillet, Diffusivity measurement of semi-transparent media: model of the coupled transient heat transfer and experiments on glass, silica glass and zinc selenide, Int. J. Heat Mass Transf. 47 (2004) 477-487, http://dx.doi.org/10.1016/j.ijheatmasstransfer.2003.07.003.

[11] R. Coquard, D. Rochais, D. Baillis, Conductive and radiative heat transfer in ceramic and metal foams at fire temperatures, Fire Technol. 48 (2012) 699732, http://dx.doi.org/10.1007/s10694-010-0167-8.
[12] M. Loretz, R. Coquard, D. Baillis, E. Maire, Metallic foams: radiative properties/comparison between different models, J. Quant. Spectrosc. Radiat. Transf. 109 (2008) 16-27, http://dx.doi.org/10.1016/j.jqsrt.2007.05.007.

[13] D. Poulikakos, K. Boomsma, On the effective thermal conductivity of a threedimensionally structured fluid-saturated metal foam, Int. J. Heat Mass Transf. 44 (2001) 827-836, http://dx.doi.org/10.1016/S0017-9310(00)00123-X.

[14] V. Leroy, B. Goyeau, J. Taine, Coupled upscaling approaches for conduction, convection, and radiation in porous media: theoretical developments, Transp. Porous Media 98 (2013) 323-347, http://dx.doi.org/10.1007/s11242-0130146-X.

[15] Taik Young Kim, Seung Wook Baek, Analysis of combined conductive and radiative heat transfer in a two-dimensional rectangular enclosure using the discrete ordinates method, Int. J. Heat Mass Transf. 34 (1991) 2265-2273, http://dx.doi.org/10.1016/0017-9310(91)90052-G.

[16] M.M. Razzaque, J.R. Howell, D.E. Klein, Coupled radiative and conductive heat transfer in a two-dimensional rectangular enclosure with gray participating media using finite elements, J. Heat Transf. 106 (1984) 613-619.

[17] C.E. Siewert, J.R. Thomas Jr., A computational method for solving a class of coupled conductive-radiative heat transfer problems, J. Quant. Spectrosc. Radiat. Transf. 45 (1991) 273-281.

[18] C.E. Siewert, An improved iterative method for solving a class of coupled conductive-radiative heat-transfer problems, J. Quant. Spectrosc. Radiat. Transf. 54 (1995) 599-605, http://dx.doi.org/10.1016/0022-4073(95)00111W.

[19] A. Ortona, C. D’Angelo, S. Gianella, D. Gaia, Cellular ceramics produced by rapid prototyping and replication, Mater. Lett. 80 (2012) 95-98, http://dx.doi.org/ 10.1016/j.matlet.2012.04.050.

[20] M.F. Modest, Radiative Heat Transfer, Academic Press, 2013.

[21] X. Fu, R. Viskanta, J.P. Gore, A model for the volumetric radiation characteristics of cellular ceramics, Int. Commun. Heat Mass Transf. 24 (1997) 1069-1082, http://dx.doi.org/10.1016/S0735-1933(97)00101-2.

[22] J.T. Farmer, J.R. Howell, Comparison of Monte Carlo strategies for radiative transfer in participating, Media (1998), http://dx.doi.org/10.1016/S0065-2717 (08)70243-0.

[23] A. Haselbacher, F.M. Najjar, J.P. Ferry, An efficient and robust particlelocalization algorithm for unstructured grids, J. Comput. Phys. 225 (2007) 2198-2213, http://dx.doi.org/10.1016/j.jcp.2007.03.018.

[24] W.H. Press, S.A. Teukolsky, W.T. Vetterling, B.P. Flannery, Numerical Recipes in C++ - The Art of Scientific Computing, Cambridge University Press, 2001.

[25] H.D. Baehr, K. Stephan, Waerme- Und Stoffuebertragung, Springer, Berlin, Heidelberg, 2008. 
[26] D.Y.S. Perraudin, Novel Method for Coupled Radiation-Conduction Simulations in Complex Geometries, Eidgenössische Technische Hochschule Zürich, 2014.

[27] G.D. Raithby, E.H. Chui, A finite-volume method for predicting a radiant heat transfer in enclosures with participating media, J. Heat Transf. 112 (1990) 415, http://dx.doi.org/10.1115/1.2910394.

[28] W.A. Fiveland, Discrete-ordinates solutions of the radiative transport equation for rectangular enclosures, J. Heat Transf. 106 (1984) 699, http://dx.doi.org/ $10.1115 / 1.3246741$.
[29] R. Viskanta, R.J. Grosh, Heat transfer by simultaneous conduction and radiation in an absorbing medium, J. Heat Transf. 84 (1962) 63-72.

[30] M.N. Özisik, Radiative Heat Transfer and Interaction with Conduction and Convection, John Wiley \& Sons, 1973.

[31] G.L. Vignoles, A Ortona, Numerical study of effective heat conductivities of foams by coupled conduction and radiation, Int. J. Therm. Sci. 109 (2016) 270278, http://dx.doi.org/10.1016/j.ijthermalsci.2016.06.013. 